\title{
Dynamics of Photoexcited Carriers in GaInAs/GaAs Quantum Dots
}

\author{
E. ILCZUK ${ }^{a, *}$, K.P. KORONA ${ }^{a}$, A. BABIŃsKI ${ }^{a}$ AND J. KUHL ${ }^{b}$ \\ ${ }^{a}$ Institute of Experimental Physics, Warsaw University \\ Hoża 69, 00-681 Warszawa, Poland \\ ${ }^{b}$ MPI für Festkörperforschung, Heisenbergstrasse 1, 70569 Stuttgart, Germany
}

\begin{abstract}
We present photocurrent and time-resolved photoluminescence investigations of $\mathrm{AlGaAs} / \mathrm{GaInAs} / \mathrm{GaAs}$ structures containing GaInAs/GaAs self-assembled quantum dots. The high electrical field in those devices significantly influences carrier dynamics. The photocurrent spectra show a double peak with maxima at 1.40 and $1.47 \mathrm{eV}$ (at $80 \mathrm{~K}$ ). These maxima are due to the GaInAs wetting layer (higher) and the quantum dots (lower). The photoluminescence spectra comprise weak excitonic luminescence from GaAs at $1.504 \mathrm{eV}\left(\right.$ at $80 \mathrm{~K}$ ) and stronger and broad emission from the $\mathrm{Ga}_{0.4} \mathrm{In}_{0.6} \mathrm{As}$ quantum dots. At $300 \mathrm{~K}$, the quantum dots emission has a lifetime of $1.1 \mathrm{~ns}$ and has a maximum at an energy of $1.38 \mathrm{eV}$. By analysis of both experiments, we can separate the influence of different radiative and nonradiative recombination processes. So, the tunneling rate: $r_{\mathrm{T}}=0.5 \mathrm{~ns}^{-1}$ and the radiative recombination rate in the quantum dots: $r_{\mathrm{RQD}}=0.4 \mathrm{~ns}^{-1}$ have been determined. The high tunneling probability (due to the influence of the built-in electric field) reveals that the tunneling effect is important for the recombination and transport processes in our structures.
\end{abstract}

PACS numbers: 72.40.+w, 78.47.+p, 78.67.-n

\section{Introduction}

Quantum dots (QDs) are quasi zero-dimensional semiconductor structures, which may find attractive applications in electronic and optoelectronic devices. Localization of an electron-hole $(\mathrm{e}-\mathrm{h})$ pair in three dimensions leads to a more efficient radiative recombination. On the other hand, the low density of states in QDs is the reason of the state filling effect observed in QDs. This results in

${ }^{*}$ corresponding author; e-mail: eilczuk $@_{\text {poczta.wp.pl }}$ 
a complicated carrier relaxation scenario reported by several groups measuring time-resolved spectroscopy [1-5].

The situation is often more complicated because the QD structures contain usually barrier and buffer layers necessary to improve the electrical characteristics of the structures. This stack of layers can unintentionally create an electrical field. High electric field in those devices influences quantum states within the QDs and can induce changes in the carrier dynamics [4].

Here, we present photocurrent (PC) and time-resolved photoluminescence (TRPL) investigations of the AlGaAs/GaInAs/GaAs field-effect structures containing self-assembled quantum dots. The TRPL signal results from carriers which recombine radiatively, its time evolution, however, is determined by the carrier decay due to both radiative and nonradiative recombination processes. On the other hand, the PC signal originates from the escaping carriers (nonradiative recombination). The complementary character of both experiments allows for a full analysis of the carrier behavior.

\section{Samples and experiment}

Samples studied in this work were grown on a [100] semi-insulating GaAs substrate using low pressure metal organic vapor phase epitaxy. The InGaAs/GaAs structures have been grown at the Australian National University. The $\mathrm{Ga}_{0.4} \mathrm{In}_{0.6} \mathrm{As}$

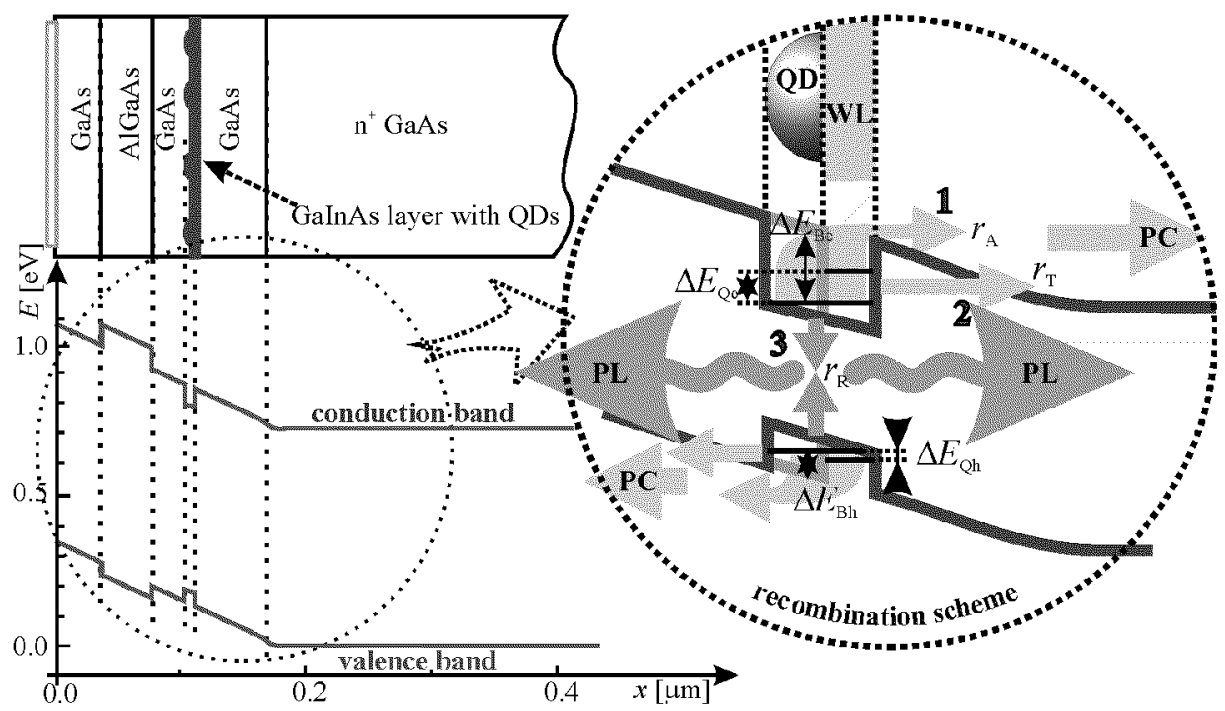

Fig. 1. Chemical structure of the sample, calculated band structure, and proposed recombination scheme. Three main recombination paths for carriers excited in the QDs are: (1) thermal escape over the GaInAs/GaAs barrier, (2) escape by tunneling and (3) radiative recombination. 
QDs were grown in the Stranski-Krastanow mode. It was found (by means of atomic force microscopy [6]) that lens shaped islands with an average basal diameter of $34 \pm 5 \mathrm{~nm}$ were formed with a surface density equal to $7.1 \times 10^{9} \mathrm{~cm}^{-2}$. The $\mathrm{Ga}_{0.4} \mathrm{In}_{0.6} \mathrm{As}$ layers were covered with undoped GaAs spacers, $\mathrm{Al}_{0.2} \mathrm{Ga}_{0.8} \mathrm{As}$ barriers, and GaAs caps (see Fig. 1). At the top of the samples, semitransparent $\mathrm{Ni} / \mathrm{Cr}$ metal contacts (forming Schottky barriers) were evaporated. A reference sample, without QDs, has been measured for comparison.

The PC has been excited by continuous monochromatic light and detected by a sensitive picoamperemeter. The measurements have been performed in a cryostat, in the temperature range from $80 \mathrm{~K}$ up to $300 \mathrm{~K}$. In the TRPL measurements, 150 fs pulses from a Ti:sapphire laser have been used for photoexcitation of free electron-hole pairs in the sample. The spectral and temporal distribution of the PL was analyzed by a monochromator and a streak camera.

\section{Results and discussion}

The signal recorded at low temperature (below $20 \mathrm{~K}$ ) comprises a sharp line of excitons in the GaAs barrier at $1.512 \mathrm{eV}$ [7], the GaAs free-electron to carbon acceptor transition at $1.494 \mathrm{eV}$ [8] and a much broader emission from the $\mathrm{Ga}_{0.4} \mathrm{In}_{0.6} \mathrm{As} / \mathrm{GaAs}$ QDs (observed in the range of 1.3-1.5 eV). At low density of QDs, it is possible to distinguish individual states [1, 2]. Unfortunately, the relatively small distances [6] between dots in our samples lead to significant interactions between the dots. So instead of the individual states, we observe one broad band of QD emission. The GaAs excitonic and QDs luminescence can be distinguished even at room temperature (see Fig. 2). The QDs emission has a fast-decaying high-energy wing and a low-energy tail. The tail persists significantly longer (for example: $\tau=1.1 \mathrm{~ns}$ at $80 \mathrm{~K}$ ).

The photocurrent spectra show a signal due to the GaAs band-to-band excitation but also double peaks attributed to the GaInAs. At $80 \mathrm{~K}$ these peaks occur at 1.40 and $1.47 \mathrm{eV}$. The lower peak is probably due to QDs. We can compare its energy with the PL peak energy at $80 \mathrm{~K}: \hbar \omega=1.38 \mathrm{eV}$. The energies are very similar, the small shift of $0.02 \mathrm{eV}$ can be explained by carrier relaxation in the electric field (the confined Stark effect). The higher peak (at $1.47 \mathrm{eV}$ ) comes from the wetting layer (WL).

Photoluminescence of the WL is very weak. In fact, we observe only a short-living $(0.1 \mathrm{~ns})$ PL signal which has a time-integrated intensity at least an order of magnitude lower than the time-integrated intensity of the QDs peak. This behavior is due to fast transfer of carriers from the WL into the QDs and due to their low radiative recombination rate, $r_{\mathrm{RWL}}$. At high temperature $r_{\mathrm{RWL}}$ is small because in $2 \mathrm{D}$ structure only a small part of excitons has $k$ vector matching to the $k$ vector of photon [5]. Moreover we expect in-plane electric fields resulting from a nonuniform charge distribution due to QDs. These fields separate electrons and holes in the WL decreasing probability of the radiative recombination. 


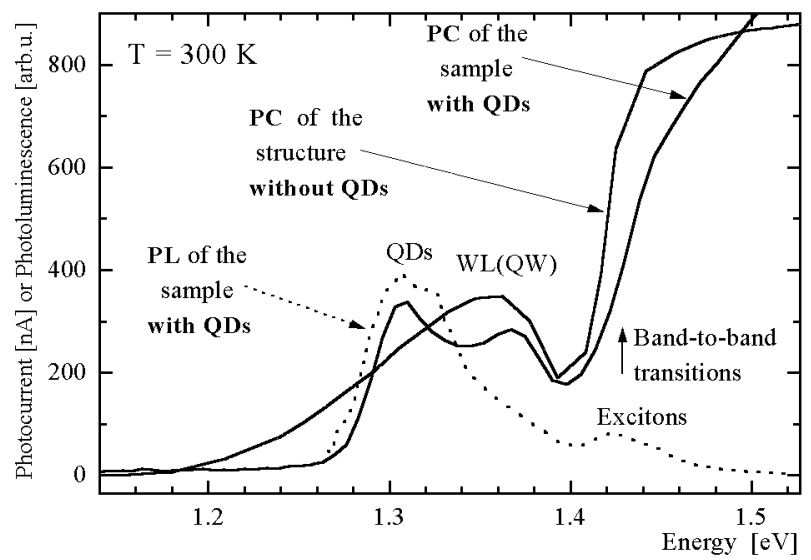

Fig. 2. Comparison of photocurrent (PC) and photoluminescence (PL) spectra of different samples at room temperature. Peaks due to quantum dots (QDs) can be observed in PC and in PL. Moreover, the PC spectra show a peak due to the wetting layer (WL) which is an analogue to the peak corresponding to the quantum well (QW) in the sample without QDs.

The low radiative recombination rate slightly influences PC signal. The PC signal from the wetting layer is strong and slightly increases at higher temperature (as the result of thermally activated delocalization of excitons from the QDs to the WL). It is expected that this delocalization process should be thermally activated with the activation energy equal to the energy difference between the WL and the QDs states, $\Delta E=E_{\mathrm{WL}}-E_{\mathrm{QD}}=60 \mathrm{meV}$. However, we have found experimentally that the activation energy of this process is very small $\left(E_{\mathrm{A}}=0 \pm 2 \mathrm{meV}\right)$. Another sample, studied for comparison, has $\Delta E=80 \mathrm{meV}$ and $E_{\mathrm{A}}=3 \mathrm{meV}$. It was suggested [9] that this effect is probably due to a competing tunneling process. A similar analysis shows that the ratio of the photocurrents excited via the $\mathrm{WL}$ and the band-to-band transition also has an activation energy, $E_{\mathrm{A}}<2 \mathrm{meV}$, much lower (than expected). In the case of the WL, we can compare the sample with QDs and the sample without QDs. For the latter sample, $E_{\mathrm{A}}=8 \pm 2 \mathrm{meV}$, has been observed. This energy is higher than in the case of the QDs sample, however still much lower than expected $(\Delta E=70 \mathrm{meV})$.

The detailed analysis of the temperature dependences is shown in Fig. 3. Since the magnitude of the photocurrent depends on many factors, we have calculated a normalized $\mathrm{PC}$ amplitude, which is equal to the ratio of the $\mathrm{PC}$ of QDs to the $\mathrm{PC}$ of the band-to-band transition $(E>1.5 \mathrm{eV})$. It can be noticed that changes of the normalized PC amplitude are rather small. It can be explained only if we take into account that the escape of carriers from the QDs is dominated by a temperature independent process. The most probable process is tunneling of $\mathrm{e}-\mathrm{h}$ pairs out of the QDs. The tunneling is possible due to the high electric field 

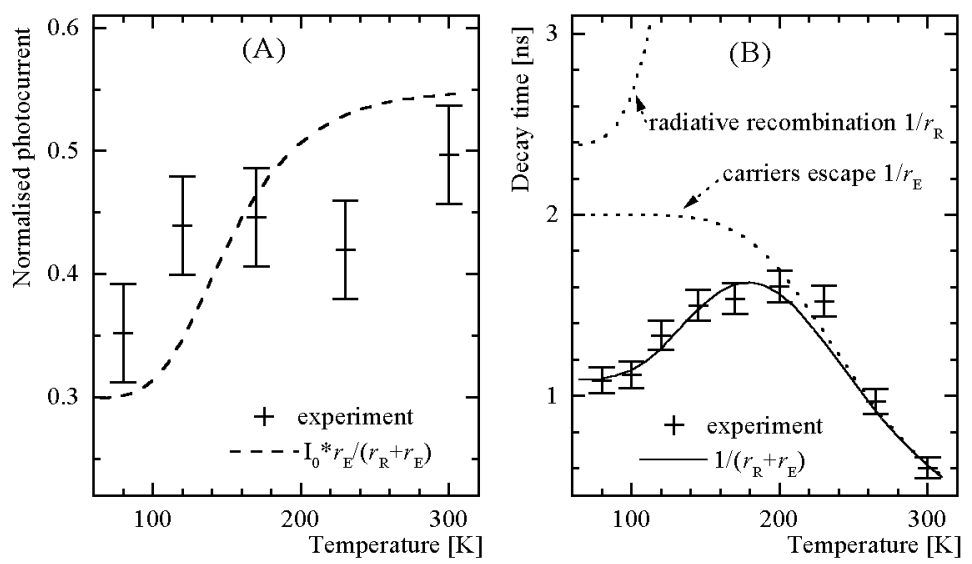

Fig. 3. Temperature dependence of: (a) Amplitudes of the QDs photocurrent (normalized, so the current of the band-to-band transition is equal to 1), the dashed curve shows the calculated dependence; (b) The PL decay times, the calculated temperature dependence of the decay times associated with radiative recombination and escape of carriers are shown as dotted lines.

present in this multilayer structure.

The photoexcited carriers trapped in the dots can either (see the recombination scheme plotted in Fig. 1): (1) escape over the barrier with a rate $r_{\mathrm{A}}$, (2) escape by tunneling to the buffer $\left(\right.$ rate $\left.r_{\mathrm{T}}\right)$ or $(3)$ recombine radiatively $\left(\right.$ rate $\left.r_{\mathrm{R}}\right)$. We neglect all the nonradiative recombination processes inside the QDs (nontransport processes) such as Auger recombination or recombination on defects inside QDs. A similar model for carrier escape leading to a photocurrent has been presented for example in Ref. [9]. The sum of the transport processes (1) and (2) gives an escape rate: $r_{\mathrm{E}}=r_{\mathrm{A}}+r_{\mathrm{T}}$. The tunneling is not strongly temperature dependent, so we assume $r_{\mathrm{T}}(T)=$ const. On the other side, the escape over the barrier is thermally activated and thus strongly depends upon temperature

$$
r_{\mathrm{A}}(T)=r_{\mathrm{A}}(T \rightarrow \infty) \exp \left(-\frac{E_{\mathrm{A}}}{k T}\right),
$$

where $r_{\mathrm{A}}(T \rightarrow \infty)$ is the escape rate at infinite temperature and $E_{\mathrm{A}}$ is an activation energy. For electrons, this energy should be equal to the height of the barrier between an electron state in a QD and the GaAs conduction band. So, for electrons: $E_{\mathrm{A}}=E_{\mathrm{Be}}$, however, the escaping electron is attracted by a hole which is present in the QD. (Numerical modeling of the carrier transport in our structure [4] shows that after the photoexcitation, the QDs are loaded with electrons and with holes.) The probability of simultaneous electron and hole escape is proportional to the product of the escape probabilities for electrons and holes. Thus this process will have an activation energy: $E_{\mathrm{A}}=E_{\mathrm{Be}}+E_{\mathrm{Bh}}$. This sum of energies equals to the difference, $E_{\mathrm{B}}$, between the GaAs energy gap and the energy emitted via radiative 
recombination of e-h pairs in the QDs. We can take this energy from the optical spectra: $E_{\mathrm{B}}=0.13 \mathrm{eV}$.

During the PC measurements, the illumination intensity is constant. Under steady-state conditions, $\nu_{\mathrm{eh}} \mathrm{e}-\mathrm{h}$ pairs are excited in the QDs per unit of time and the steady-state occupation number of e-h pairs is equal to $n_{\mathrm{eh}}=\nu_{\mathrm{eh}} /\left(r_{\mathrm{R}}+r_{\mathrm{E}}\right)$ (here $\tau=1 /\left(r_{\mathrm{R}}+r_{\mathrm{E}}\right)$ is the $\mathrm{e}-\mathrm{h}$ lifetime). The photocurrent is proportional to the number of escaping carriers: $2 n_{\mathrm{eh}} r_{\mathrm{E}}$. Thus the PC is given by an equation: $I_{\mathrm{PC}}=\alpha \nu_{\mathrm{eh}} r_{\mathrm{E}} /\left(r_{\mathrm{R}}+r_{\mathrm{E}}\right)=\alpha \nu_{\mathrm{eh}}\left(r_{\mathrm{A}}+r_{\mathrm{T}}\right) /\left(r_{\mathrm{R}}+r_{\mathrm{A}}+r_{\mathrm{T}}\right)(\alpha$ is a constant $)$. Since $I_{\mathrm{PC}}$ depends on three recombination processes, we also need information about $r_{\mathrm{R}}$ in order to describe the PC.

On the other hand, the PL decay time, $\tau$ depends on the radiative recombination and the escape of $\mathrm{e}-\mathrm{h}$ pairs, according to an equation: $\tau=1 /\left(r_{\mathrm{R}}+r_{\mathrm{A}}+r_{\mathrm{T}}\right)$. The PL transients measured by TRPL show that the PL lifetime initially increases with temperature. It obtains its maximum value at about $200 \mathrm{~K}$. Then, at higher temperatures it decreases. This gives interesting information about recombination processes. However, in order to understand the effect of the lifetime increase, we should notice that the PC signal from WL becomes stronger at higher temperature. From these two facts, we conclude that at high temperature, delocalization of excitons from QDs to WL occurs. Since the radiative recombination from the WL state is very small, thermally induced occupation of this state leads to a decrease in the radiative recombination rate. The $\mathrm{e}-\mathrm{h}$ pair distribution will depend on the ratio of density of states in the wetting layer $\left(D_{\mathrm{WL}} \times T\right)$ in comparison to that of the QDs $\left(N_{\mathrm{QD}}\right): D_{\mathrm{WL}} T / N_{\mathrm{QD}}(=A T)$ and on the difference of energies between a state in the WL $\left(E_{\mathrm{WL}}\right)$ and in the $\mathrm{QDs}\left(E_{\mathrm{QD}}\right)$. So, the ratio of the e-h pairs concentration in the WL $\left(n_{\mathrm{WL}}\right)$ to the concentration in the QDs $\left(n_{\mathrm{WL}}\right)$ is equal to

$$
\frac{n_{\mathrm{WL}}}{n_{\mathrm{QD}}}(T)=A T \exp \left(-\frac{E_{\mathrm{WL}}-E_{\mathrm{QD}}}{k T}\right) \text {. }
$$

In real QDs the radiative recombination rate $r_{\mathrm{QD}}$ is temperature independent [3]. The temperature dependence of the radiative recombination rate for $2 \mathrm{D}$ structures is reciprocally proportional to temperature [5], $r_{\mathrm{RWL}}(T)=\gamma_{\mathrm{WL}} / T$. Taking this into account, we can calculate that the effective radiative recombination rate, $r_{\mathrm{R}}(T)$, is equal to

$$
r_{\mathrm{R}}(T)=\frac{r_{\mathrm{RQD}}+\gamma_{\mathrm{RWL}} A \exp \left(-\frac{E_{\mathrm{WL}}-E_{\mathrm{QD}}}{k T}\right)}{1+A T \exp \left(-\frac{E_{\mathrm{WL}}-E_{\mathrm{QD}}}{k T}\right)} .
$$

For the calculation procedure, we have taken the energy difference $E_{\mathrm{WL}}-E_{\mathrm{QD}}=60 \mathrm{meV}$, as measured by the $\mathrm{PC}$ experiment. The radiative recombination from the WL is very small, so it can be neglected: $\gamma_{\mathrm{RWL}}=0$. It is more convenient to introduce an escape lifetime and a radiative lifetime instead of the escape rate and radiative recombination rate, respectively. The calculated temperature dependence of the radiative lifetime $\left(\tau_{\mathrm{R}}=1 / r_{\mathrm{R}}\right)$ and the 
escape lifetime $\left(\tau_{\mathrm{E}}=1 / r_{\mathrm{E}}\right)$ are plotted in Fig. 3. The effective lifetime (also in Fig. 3) is equal to $\tau=1 /\left(1 / \tau_{\mathrm{R}}+1 / \tau_{\mathrm{E}}\right)$. As results of the fitting and calculation, we have found: the tunneling ratio, $r_{\mathrm{T}}=0.50(5) \mathrm{ns}^{-1}$, the activated escape ratio, $r_{\mathrm{A}}(T \rightarrow \infty)=170 \mathrm{~ns}^{-1}$, and the $\mathrm{QDs}$ radiative recombination ratio, $r_{\mathrm{RQD}}=0.42(5) \mathrm{ns}^{-1}$.

The thermally activated escape-over-barrier ratio, $r_{\mathrm{A}}$, is negligible at low temperatures. However, at $T>200 \mathrm{~K}$ it becomes important. Based on Eq. (1) we calculated that at $T=200 \mathrm{~K}$ the escape-over-barrier ratio is $r_{\mathrm{A}}(T)=0.09 \mathrm{~ns}^{-1}$. So, the tunneling ratio $\left(r_{\mathrm{T}}=0.5 \mathrm{~ns}^{-1}\right)$ is much higher than the $r_{\mathrm{A}}$ in the $T<200 \mathrm{~K}$ range. We can conclude that the tunneling effect is very important for recombination and transport processes in our structures. The tunneling process is probably so efficient since the higher electric field forms a gradient of the potential. In the gradient, the barriers become triangular, thin, and transparent for the tunneling.

According to our calculations, the increase in the lifetime with temperatures up to $200 \mathrm{~K}$ is an effect of a significant increase in the radiative lifetime (see upper dotted curve in Fig. 3b), while the escape lifetime remains constant in this temperature range (lower dotted curve in Fig. $3 \mathrm{~b}$ ).

\section{Conclusions}

We have presented photocurrent and time-resolved photoluminescence investigations of $\mathrm{AlGaAs} / \mathrm{InGaAs} / \mathrm{GaAs}$ structures containing self-assembled $\mathrm{Ga}_{0.4} \mathrm{In}_{0.6} \mathrm{As} / \mathrm{GaAs}$ quantum dots. Different (competitive) recombination processes influence both the PL and the PC signals, however, in different (partially opposite) ways. Therefore, by analysis of both experiments, we can distinguish the influence of different (radiative and nonradiative) recombination processes. We have observed (i) small changes of the PC amplitude vs. temperature that indicates strong carrier transport by tunneling and (ii) a complex dependence of the PL lifetime, which indicates that a few recombination processes are involved. Based on both experiments, the tunneling rate: $r_{\mathrm{T}}=0.5 \mathrm{~ns}^{-1}$ and the QDs radiative recombination rate: $r_{\mathrm{RQD}}=0.4 \mathrm{~ns}^{-1}$ have been found. Such a high tunneling probability means that the tunneling effect is very important for recombination and transport processes in our structures. The tunneling process is probably so efficient since the higher electric field forms a gradient of the potential leading to triangular barriers.

\section{Acknowledgments}

K. P. K. gratefully acknowledges financial support (Forschungsstipendium) by the Alexander-von-Humboldt Foundation. This work was supported in part by the State Committee for Scientific Research (Poland), grant no. 2 P03B 04318. 


\section{References}

[1] F. Adler, M. Geiger, A. Bauknecht, F. Scholz, H. Schweizer, M.H. Pilkuhn, B. Ohnesorge, A. Forchel, J. Appl. Phys, 80, 4019 (1996).

[2] S. Grosse, J.H.H. Sandmann, G. von Plessen, J. Feldmann, H. Lipsanen, M. Sopanen, J. Tulkki, J. Ahopelto, Phys. Rev. B 55, 4473 (1997).

[3] A. Kurtenbach, W.W. Rühle, K. Eberl, Solid State Commun. 96, 265 (1995).

[4] K.P. Korona, A. Babiński, J. Kuhl, J.M. Baranowski, R. Leon, submitted to Phys. Status Solidi A.

[5] J. Feldmann, G. Peter, E.O. Göbel, P. Dawson, K. Moore, C. Foxon, R.J. Elliott, Phys. Rev. Lett. 59, 2337 (1987).

[6] A. Babiński, K.P. Korona, J.M. Baranowski, in: Proc. 11th Int. Semiconducting and Insulating Materials Conf. (XI SIMC), Canberra 2000, Eds. C. Jagadish, N.J. Welham, IEEE Publishing, Piscataway (NJ) 2001, p. 232.

[7] J.A. Rossi, C.M. Wolfe, G.E. Stillman, J.O. Dimmock, Solid State Commun. 8, 2021 (1970).

[8] B.J. Skromme, T.S. Low, T.J. Roth, G.E. Stillmann, J.K. Kennedy, J.K. Abrokwah, J. Electron. Mater. 12, 433 (1983).

[9] W.-H. Chang, T.M. Hsu, C.C. Huang, S.L. Hsu, C.Y. Lai, N.T. Yeh, T.E. Nee, J.-I. Chyi, Phys. Rev. B 62, 6959 (2000). 